\title{
Legal and practical issues at the end of life
}

\author{
Author: Catherine Wills ${ }^{\mathrm{A}}$
}

KEYWORDS: Legal, capacity, nominated persons, DNACPR, communication

\section{Introduction}

Treating patients who are approaching the end of their life is a complex and sensitive matter. The GMC guidance Treatment and care towards the end of life: good practice in decision making ${ }^{1}$ offers advice to doctors on how to care for a patient approaching the end of their life. It classifies patients as 'approaching the end of life' when they are likely to die within the next 12 months. This includes patients whose death is imminent, as well as those with advanced progressive incurable conditions, general frailty and conditions that mean they are expected to die within 12 months, existing conditions if they are at risk of dying from a sudden acute crisis in their condition and life threatening acute conditions caused by sudden catastrophic events.

\section{Breaking bad news}

Breaking bad news is an important and complex communication task. As with any aspect of practice, doctors must recognise and work within their competence and should take steps to improve the quality of their work, taking part in activities that maintain and develop competence and performance. The authors of a paper looking at this issue concluded that for many clinicians additional training in breaking bad news could be useful. ${ }^{2}$ The researchers proposed a strategy for breaking bad news known as SPIKES, suggesting it might help increase doctors' confidence and encourage patient participation in difficult treatment decisions. The six steps are summarised below and may be useful to adopt whenever bad news needs to be conveyed.

Set up - think about what you want to say in advance and try to arrange it so that your discussion can take place in a quiet, uninterrupted setting at a time when you will not need to rush away. Arrange for privacy and consider suggesting that the patient is accompanied by a friend or relative. Make sure there is sufficient seating in the room and turn off or mute any electronic devices so that the patient has your full attention. Make a connection with a patient, for example by maintaining eye contact to establish rapport.

Author: ${ }^{\text {A }}$ deputy head of advisory services, Medical Defence Union
Perception - Implementing the axiom 'before you tell, ask', use open-ended questions to find out how the patient perceives the situation to help you tailor how you deliver the information and where to begin. The understanding of patients and their family members may differ and they may also need time to process the information. Patients may provide you with an opening to begin discussing their condition or they may try to avoid hearing what you are saying. You will need to gauge your approach accordingly.

Invitation - Most patients express a desire for full information but some patients may shun information as a coping mechanism. If patients do not want to hear details, offer to answer any questions they might have in future or to talk to their relative or friend. Use language appropriate to the patient's level of education and understanding. Reflecting the patient's words and body language may be helpful to put the patient at ease and to make sure that the patient understands the discussion. Avoid euphemisms and jargon. Tune into the patient's reaction so you can provide information at a pace they will understand.

Knowledge - Warning the patient that you have bad news to tell them might lessen the shock of the disclosure. Make sure the patient and their relative have enough time to absorb and react to the information and ask further questions. Provide opportunities for them to ask questions and provide reassurance that they will have ongoing support.

Empathy - Don't make assumptions about what emotions a patient is feeling and the information they might want. Observe their signs of emotion and respond with empathy. Encourage them to express their concerns and views and respect their wishes about how much information they want to hear. Remember all patients are different.

\section{Assessing capacity to make decisions}

Physicians must work on the presumption that every adult has the mental capacity to make their own decisions about care and treatment. It is important not to assume incapacity on the basis of age, behaviour, medical condition or any other factor. Remember that a patient who has mental capacity has the right to make a decision that their doctor may disagree with, or consider irrational or unwise. Making decisions that appear irrational does not, of itself, indicate a lack of mental capacity but may reflect individual preferences or values.

The GMC end-of-life guidance says that, if a patient's capacity to make a decision may be impaired:

...you must provide the patient with all appropriate help and support to maximise their ability to understand, retain, use or weigh up the information needed to make that decision or 
communicate their wishes. You must assess their capacity to make each decision, at the time it needs to be made. ${ }^{1}$

The assessment of mental capacity in England and Wales is set out in the Mental Capacity Act (MCA) 2005, and in Northern Ireland in the Mental Capacity Act (Northern Ireland), when it comes into force. In Scotland, the Adults with Incapacity Act (Scotland) 2000 covers mental capacity in adults. Bear in mind that mental capacity may fluctuate and that lack of capacity to make one decision does not necessarily mean that a person will always lack capacity to make that decision, or that the person lacks capacity to make a decision about something else. A patient should be provided with all practicable help before they are deemed to lack capacity to make their own decision. This may include support through an advocate or a translator, for example.

Even if a patient is found to lack mental capacity to make a decision, a doctor should still involve the patient as far as possible.

Some patients who lack capacity may have made an advance decision or have put in place an attorney or legal proxy to make decisions on their behalf. Otherwise, you will need to provide any treatment based on what would be of overall benefit to the patient and which option would be least restrictive of their future choices. When making decisions about overall benefit, it is important to consult with those close to the patient to help you reach a view. While not always possible with end-of-life care, if a patient lacks capacity, consider whether they are likely to regain capacity and if so, if the decision can be deferred until then.

\section{Advance care planning and advance decisions}

The GMC end-of-life guidance ${ }^{1}$ advises that when a patient in your care is facing the possibility that their mental capacity may become impaired, you should encourage them to think about what they might want for themselves if and when that happens. That would include discussion of the patient's wishes, preferences and fears about their future care, their underlying values, who they might want you to involve and what support they might need. In addition, consider if it might be helpful for the patient to make an advance decision about any specific treatment.

Some patients may want to refuse particular treatment in advance. An 'advance decision' that is made by an adult in England and Wales, and is valid and applicable, is legally binding. ${ }^{3}$

If you become aware of an advance refusal by the patient, you should assess its validity and applicability to the situation at hand. The legal framework for making this assessment varies across the UK jurisdictions. The GMC end-of-life guidance summarises this in paragraphs $70-71 .{ }^{1}$ If you find that an advance decision is not legally binding it should be taken into account as evidence of a person's wishes, for the purpose of assessing what would be of overall benefit to them.

Some patients might make an advance request for particular treatments, in which case you should explain that, while future decisions cannot be bound by their request, it will be given weight by those deciding what is in their overall benefit, if they lose capacity.

\section{Attorneys and nominated persons}

A person with capacity may nominate a lasting power of attorney $(\mathrm{LPA})^{3}$ to make healthcare decisions for them when they have lost capacity in England and Wales.
In Scotland, a person can nominate a welfare power of attorney (WPA) to make decisions, including those about medical treatment.

In Northern Ireland the Mental Capacity Act (Northern Ireland) 2016 will, when it comes into force, enable a patient to specify a nominated person whose views will need to be taken into account when decisions are made about what would be in the patient's best interest when they do not have capacity to decide for themselves.

You should aim to reach consensus about what treatment would be of overall benefit to a patient who lacks capacity. However, where there is disagreement, it may be helpful to obtain a second opinion or to involve others such as independent advocates or mediators. In some cases, it may be necessary to obtain legal advice and/or a ruling from the relevant statutory body or court.

\section{DNACPR decisions}

The GMC says that 'if cardiac or respiratory arrest is expected as part of the dying process, making and recording an advance decision not to attempt CPR will help ensure the patient dies in a dignified and peaceful manner'. The Resuscitation Council UK ${ }^{4}$ advises that decisions about CPR should be considered, discussed and recorded as an element of care of people recognised by healthcare professionals as approaching the end of their lives (ie within the last year of life).

Discussion about CPR may be less difficult if it forms part of a broader discussion about the patient's wishes. You should not force discussion or information onto patients but should offer opportunities to discuss and must provide information to those who want it.

Both the $\mathrm{GMC}^{1}$ and the Resuscitation Council UK ${ }^{4}$ provide guidance on making DNACPR decisions.

\section{Respecting patient confidentiality}

The GMC guidance Confidentiality: good practice in handling patient information ${ }^{5}$ states that doctors must be considerate to those close to the patient and sensitive and responsive in giving them information and support, while respecting the patient's confidentiality (paragraph 34). If a patient lacks capacity to make a decision, it is reasonable to assume that the patient would want those close to them to be kept informed. But, if possible, establish what information the patient wants you to share with those close to them and in what circumstances, while they have capacity in advance of their condition deteriorating. This can help avoid disclosures that patients might object to. NICE guidance (NG31) on Care of dying adults in the last days of life ${ }^{6}$ includes recommendations for how health professionals can establish that a person is nearing the end of life and how to establish their communication needs and expectations.

\section{Artificial nutrition and hydration}

Patients and those close to them may consider nutrition and hydration administered through a tube or drip to be part of basic nurture but this is regarded in law as medical treatment. Doctors are expected to explain the 'benefits, burdens and risks' to patients and those close to them and make it clear that nutrition and hydration will always be offered where it is considered to be of overall benefit to a patient. However, if a decision is taken not 
to provide clinically assisted nutrition and hydration, patients and those close to them should be reassured the patient will continue to receive high quality care, with any symptoms addressed.

If considering withdrawing nutrition or hydration from a patient in a persistent vegetative state (PVS), the courts in England, Wales and Northern Ireland require that they are approached for a ruling. This is not specified in Scotland. However, in a recent legal ruling in the case of a woman with Huntingdon's disease known as ' $M$ ', the Court of Protection in England and Wales found that as long as doctors and relatives of the patient agree and medical guidelines are followed, there is no need to obtain judicial authorisation. The decision is likely to be appealed. Your medical defence organisation and trust/health board legal department can provide advice in individual cases.

\section{References}

1 General Medical Council. Treatment and care towards the end of life: good practice in decision making. London: GMC, 2010.
2 Baile WF, Buckman R, Lenzi R et al. SPIKES-A six-step protocol for delivering bad news: application to the patient with cancer. Oncologist 2000;5:302-11.

3 Office of the Public Guardian. Mental Capacity Act 2005: code of practice. The Stationery office, 2005.

4 Smith G, Pitcher D. Prevention of cardiac arrest and decisions about CPR. London: Resuscitation Council UK, 2015.

5 General Medical Council. Confidentiality: good practice in handling patient information. London: GMC, 2017.

6 National Institute for Health and Care Excellence. Care of dying adults in the last days of life. NICE guideline [NG31]. London: NICE, 2015.
Address for correspondence: Dr Catherine Wills, MDU Services Limited, One Canada Square, London, E14 5GS.

Email: media@themdu.com

\section{NGC

\section{New to systematic reviewing, or need to improve your critical appraisal skills?}

Our intensive 1-day courses will introduce you to the key principles of critical appraisal and systematic reviewing, and equip you with the basic skills to put your knowledge into practice.

$>$ Introduction to critical appraisal > Systematic reviews and meta-analysis in action

Discount available if both courses are booked together.

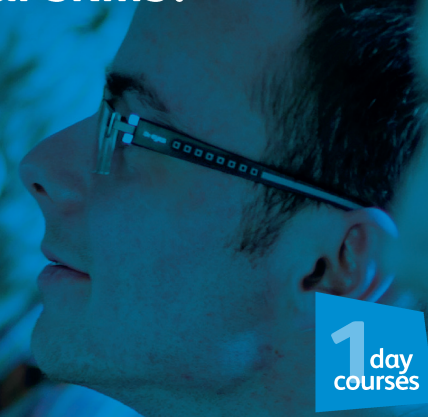

\section{'Informative and practical'} Dr Rajeswari Ramaraj
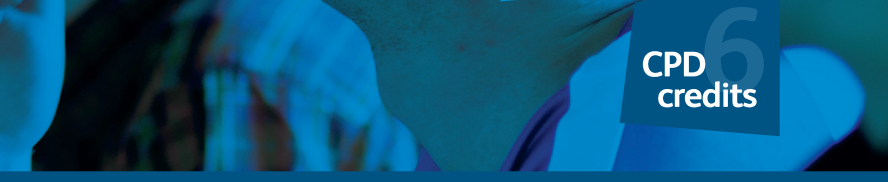

For course dates and more information visit: www.ngc.ac.uk/training-courses 